\title{
TTR
}

Traduction, terminologie, re?daction

\section{Rappel à l'ordre cartésien : Alejo Carpentier en traduction française}

\section{Enriqueta Ribé}

Volume 2, numéro 1, 1er semestre 1989

Carrefours de la traduction

URI : https://id.erudit.org/iderudit/037037ar

DOI : https://doi.org/10.7202/037037ar

Aller au sommaire du numéro

Éditeur(s)

Association canadienne de traductologie

ISSN

0835-8443 (imprimé)

1708-2188 (numérique)

Découvrir la revue

Citer cet article

Ribé, E. (1989). Rappel à l'ordre cartésien : Alejo Carpentier en traduction française. TTR, 2(1), 117-123. https://doi.org/10.7202/037037ar d'utilisation que vous pouvez consulter en ligne.

https://apropos.erudit.org/fr/usagers/politique-dutilisation/ 


\section{Rappel à l'ordre cartésien: Alejo Carpentier en traduction française}

\section{Enriqueta Ribé}

J'analyserai ici un texte qui pourrait s'interpréter comme une traduction hypertextuelle, mais qui est en réalité un bon exemple de censure traduisante. Il s'agit du chapitre II du roman le Recours de la Méthode (Carpentier, 1974, pp. 37-65).

Nous sommes devant un récit à la troisième personne où le narrateur raconte le retour du Premier Magistrat dans son pays, en provenance de Paris, dans le but d'étouffer la première d'une série de révoltes contre son gouvernement.

Deux heures après leur arrivée dans leur suite du Waldorf Astoria... (Carpentier, 1975a, p. 37)

Le récit se poursuit toujours à la troisième personne:

Et comme rien ne pressait ce soir-là, le Premier Magistrat, très amateur de grand opéra, voulut entendre un Pelléas et Mélisande qui était à l'affiche du Metropolitan Opera House... (Carpentier, 1975a, p. 16)

C'est à partir d'ici que les problèmes commencent. Il nous faudra donc comparer les textes espagnol et français pour dégager le sens des transformations que le traducteur fait subir au texte-source.

... se sentaron pues, en primera fila, alzó su batuta el director, y una enorme orquesta que tenían ahi, a sus pies, empezó a no sonar.
... Ils s'assirent donc, au premier rang, le chef d'orchestre leva sa baguette, et l'orchestre énorme qui était à leurs pieds commença à faire semblant de jouer. 
Y ahora, - cambio de decoración una señora medieval con acentos de Kansas City... Ahora, el espectáculo de las galerías y corredores suscitó al P. M. algunas divertidas y punzantes observaciones.

(Carpentier, 1975b, pp. 38-39)
Maintenant - changement de décor une dame en costume moyenâgeux lit une lettre avec l'accent de Kansas City... Voici à présent le spectacle du foyer et des couloirs qui lui inspire des remarques amusées et piquantes.

(Carpentier, 1975a, pp. 38-39)

On n'a toujours pas quitté la troisième personne, mais le lecteur est sollicité par des indicateurs d'allocuté qui suggèrent le «tú»: «se sentaron pues», "ahi a sus pies» y «ahora», «Ahora, el espectáculo».

Ces indicateurs d'allocuté frappent le lecteur hispanophone parce qu'ils appartiennent aux instances de discours et non pas au registre de la narration. ${ }^{1}$ Ils frapperaient aussi le lecteur francophone s'ils avaient été conservés, mais nous ne trouvons pas «là», «à leurs pieds", ni «maintenant, le spectacle», formulations qui insistent sur le «maintenant, changement de décor» conservé dans la traduction.

La narration se poursuit à la troisième personne jusqu'à ce que, masquée, ou mieux médiatisée par une longue phrase, descriptionréflexion sur la société américaine, elle glisse à la première personne:

Aunque ciertos apellidos de añeja ascendencia holandesa o británica se les remontara al siglo XVII cobraban al sonar en las inmediaciones del Central Park un no sé qué de producto importado ... como los imprecisos títulos de Marqueses de la Real Proclamación... que nos gastábamos en América latina.

(Carpentier, 1975b, p. 39)
Certains noms de vieille souche hollandaise ou britannique remontaient il est vrai au XVIIIe siècle, mais ils prenaient, quand on les entendait aux alentours du Central Park, un je ne sais quel air de produit importé... comme les titres imprécis de Marquis de la Royale Proclamation... que nous nous décernions en Amérique latine.

(Carpentier, 1975a, p. 39)

Ce passage à la première personne du pluriel est ponctué par l'utilisation de termes qui correspondent non plus à l'instance du discours, mais aux objets «réels», aux temps et lieux «historiques»:

... el clima de la ópera que se representaba esta noche

(Carpentier, 1975b, p. 19)
... à l'égal de l'opéra que l'on jouait $c e$ soir-là...

(Carpentier, 1975a, p. 19)

"Esta» à la place de «esa", (mot qui respecterait les règles de la grammaire), faisant glisser la référence implicite au sujet parlant, est un indicateur d'allocuté. Le traducteur, embarrassé par «l'incorrection"

1. «Les instances de discours, c'est-à-dire les actes discrets et chaque fois uniques par lesquels la langue est actualisée en parole par un locuteur".

Dans cette «instance de discours» je/tu sont accompagnés d'une «série d'indicateurs» (des pronoms, des adverbes, des locutions adverbiales) "ce», «ici», «maintenant». "Ici' et 'maintenant' délimitent l'instance de discours contenant 'je'».

Pour une analyse détaillée de ce problème voir l'étude sur «la Nature des pronoms», Benveniste (1966, pp. 251-257). Les citations qui précèdent sont extraites de cet article. 
ne se gêne pas pour traduire par hypercorrection "ce soir-là", à la place de «ce soir». La surprise que le «esta» du texte espagnol produit chez le lecteur est donc annulée par le texte français.

L'écriture acquiert ainsi un calme, une sérénité qui efface l'intention de faire signe sur le sujet du discours. En effet, qui parle? Aucune mention explicite, aucun antécédent, même éloigné dans le récit, ne permet de rattacher cette expression à un «je» qui prononce "esta" (ce soir). La chaîne co-référentielle est ébranlée; le «tú» du lecteur est attiré vers le récit, mais le locuteur reste dans l'ombre, caché au regard. Après cette émergence du je-nous, le récit reprend à la troisième personne:

$Y$ se vieron luego-luego, los tres, en un apartamento de la calle $42 \ldots$

(Carpentier, 1975b, p. 40)

Quelques instants plus tard, tous trois étaient dans un appartement de la $42 \mathrm{e}$

Rue...

(Carpentier, 1975a, p. 40)

Mais ce récit à la troisième personne conserve un élément du langage familier, de la parole qui s'adresse «in visu»: "luego-luego (qui une fois encore enfreint la grammaire). Le traducteur efface à nouveau cette marque en écrivant "quelques instants plus tard" au lieu de (par exemple) «tout de suite après».

Le récit - dans les deux textes - est hésitant: première personne du pluriel, troisième, première encore, et toujours des indicateurs qui suggèrent le «tú». Ces indicateurs, qui rapprochent la narration des faits racontés, qui placent le lecteur au même point de vue que le narrateur sont gommés - pour la plupart - dans le texte français.

Dans le texte-source, le procédé tire le récit vers l'événement: ...era moda ahora, esto de mezclar licores - que les hizo establecer divertidas escalas entre lo de acá y los minyules... de nuestras tierras calientes.

Hoy, excepcionalmente, lo veía beber así su hijo Ariel...

(Carpentier, 1975b, p. 40)

Ces mélanges étaient maintenant à la mode - ce qui leur permit d'établir toute une gamme de comparaisons anusantes entre les boissons qu'on leur servait et les mint-juleps... de nos terres chaudes.

C'est la première fois que son fils Ariel le voyait boire ainsi...

(Carpentier, 1975a, p. 40)

Dans le texte d'arrivée, les expressions qui actualisent ce discours disparaissent; «acá» (ici) n'est pas retenu, et on lit «qu'on leur servait» alors qu'on aurait dû traduire equ'on leur servait ici». De même «hoy» (aujourd'hui) est traduit par "C'est la première fois". Dans les deux cas, les marques d'allocuté sont supprimées.

Puis, sur toute une page, le récit reprend la forme innocente de la troisième personne et on arrive au moment de la description du carnaval à la Havane où:

Après avoir loué un domino noir, il [le Premier Magistrat] alla au bal de Tacón, où une mulâtresse... invita le Premier Magistrat à l'accompagner dans sa chambre... (Carpentier, 1975a, pp. 42-43) 
Il faut remarquer que le narrateur emploie la troisième personne du singulier «alla», «invita». Le pluriel qui suit immédiatement, «prirent», ne peut que reprendre anaphoriquement le «il» du Premier Magistrat et le «elle» de la mulâtresse:

Ils prirent un fiacre...

(Carpentier, 1975a, p. 43)

Lorsqu'ils sont arrivés à destination, le récit fait place au dialogue, en apparence farfelu:

- Veillez sur mon sommeil, cher compère, me dit le Premier Magistrat.

- Ne vous tracassez pas, cher compère, j'ai ici ce qu'il faut, répondis-je en tirant mon browning de la poche intérieure de mon veston...

(Carpentier, 1975a, p. 43)

Et plus loin:

... Du coup je réveille le Premier Magistrat... et nous nous engageons à pied... dans la rue qui conduit au quai Saint François, où nous attend le cargo prêt à lever l'ancre... (Carpentier, 1975a, p. 44)

Ici, le «nous» ne peut être que l'anaphore du «je» (je réveille) et du «il» mis pour «le Premier Magistrat».

L'apparition de ce «je» présuppose - si l'on essaie de s'en tenir à la cohérence du récit - que le Magistrat est accompagné d'un garde du corps. Mais cette hypothèse n'est appuyée par aucune trace dans l'argumentation ou la syntaxe. Il s'est plutôt produit, semble-t-il, une brisure dans le tissu du récit. Cette brisure, ce trou, signale les points par où le récit peut se déchirer. L'émergence du «je» non co-référentiel, travaillant à l'encontre de la syntaxe, ne peut s'expliquer dans la logique du récit que comme un lapsus, ce qui est à exclure car il s'étend tout au long d'un chapitre et est soigneusement encadré d'indicateurs d'allocuté, comme nous l'avons montré. À un autre niveau, on peut l'interpréter comme une feinte de lapsus: cette incohérence apparente signale une complicité de l'auteur, une ruse destinée à authentifier le récit. C'est une façon de présenter l'histoire en train de se faire, c'est montrer la possibilité pour celui qui dit «je» de participer au «se faire» de l'histoire. Au milieu du récit fini, compact, le «je» rappelle l'inachevé, la possibilité d'intervention, le détournement possible d'événements à venir. L'émergence de l'auteur sert à provoquer l'«illusion réaliste» (Angenot, 1978, pp. 95-100). Mais il y a plus. Le langage du roman a ouvert à l'auteur la possibilité d'inaugurer un dialogue avec les lecteurs. Le roman se charge ainsi d'allusions, de références, et même d'un savoir dont la clé ne fonctionnera que pour les lecteurs qui partagent les expériences de l'auteur. Ainsi, le vrai fait irruption au milieu du vraisemblable. Mais le «vrai» échappe aux règles qui veulent se l'approprier (ici, les règles qui régissent la construction romanesque), ce qui est exprimé dans le texte au moyen de ce que nous avons appelé la feinte de lapsus. L'irruption du «je» illustre 
l'insuffisance du genre à contenir le «vrai» qui apparaît comme incohérence («anomalie» qui a, peut-être, poussé le traducteur à «améliorer» le texte) et, ce faisant, signale une complicité de l'auteur et des lecteurs-acteurs. L'irruption de l'auteur à travers les déchirures du tissu romanesque présuppose l'actualisation d'un savoir politique amené à la conscience du lecteur. On a réussi par ce tour de force à briser la distance esthétique que le genre romanesque impose entre l'auteur et le lecteur et à la dénoncer comme le siège d'un travail idéologique. Du coup, c'est la structure du roman bourgeois contemporain qui est mise en cause: cette irruption de l'auteur inscrit la transgression des limites imposées par le discours romanesque en tant que formation institutionnelle. Cette transgression illustre l'insuffisance (peut-être même l'inadéquation) de l'instrument à assurer une communication, dans le domaine de l'art, entre l'auteur et les lecteurs. La mise en évidence de cette inadéquation dénonce le roman bourgeois comme siège d'un système complexe d'occultation des rapports entre l'art et la société. Occultation fondée sur le sacro-saint principe de la distance esthétique qui impose à l'auteur de ne pas intervenir dans son roman. ${ }^{2}$

Carpentier ne respecte pas la règle de non-intervention. Il se mêle aux histoires qu'il raconte et il fait davantage: il y mêle les lecteurs. Cette intervention n'est pourtant ni moraliste ni exemplaire. Elle prend la forme du jeu; c'est sur un ton narquois qu'il faut entendre le: "Veillez sur mon sommeil, cher compère» et «j'ai ici ce qu'il faut". Comprises sur le mode d'une ironie malicieuse, les phrases se changent en critique formulée sur le mode de la plaisanterie: le personnage a l'air de bouger en liberté, mais comme il le fait dans l'enceinte de la prison que le roman lui construit, il est en réalité un personnage en liberté surveillée, une bête de cirque évoluant dans l'enceinte de l'arène.

Ce que l'auteur affirme par cette irruption, c'est le refus de signer, en dehors du corps romanesque, le livre qui peut faire accéder un nom à la célébrité, au succès, même s'il faut pour cela être chassé

2. C'est ce que $M$. Blanchot souligne en ces termes:

«Le mot d'ordre est impérieux: le romancier ne doit pas intervenir. L'auteur même si madame Bovary c'est moi - supprime tous les rapports directs entre lui et le roman; la réflexion, le commentaire, l'intrusion moralisante telle qu'elle est encore autorisée, avec éclats, chez Balzac deviennent péchés. Pourquoi? Pour deux raisons différentes quoique presque confondues. La première: ce qui est raconté a valeur esthétique dans la mesure où l'intérêt qu'on y prend est un intérêt à distance, le désintéressement - catégorie essentielle du jugement de goût depuis Kant et mème Aristote - signifie que l'acte esthétique ne doit se fonder sur aucun intérêt, s'il veut en produire un qui soit légitime: intérêt désintéressé...

L'autre raison est presque la même quoique très différente: l'auteur ne doit pas intervenir, parce que le roman est une cuvre d'art et que l'œuvre d'art existe toute seule, chose irréelle, dans le monde hors du monde, il faut la laisser libre, supprimer les détails, couper les amarres, pour la maintenir dans son statut d'objet imaginaire.» (Blanchot, 1969, p. 560). 
du corpus, s'interdire de pointer du doigt ou crier au voleur. Curieux paradoxe dont il faut chercher la correspondance dans les modalités qu'acquiert l'exercice de la liberté et de la responsabilité dans la société capitaliste.

Le mécanisme que nous venons d'analyser inscrit une contestation de ces modalités, et il l'inscrit en profondeur; c'est d'abord et surtout la structure même du roman qu'il s'agit de ronger.

Cette analyse du texte-source devient difficile, sinon impossible, dans le texte traduit, où presque tous les indicateurs d'allocuté qui préparent l'émergence du «je» ont été supprimés. On est en droit de s'interroger sur les raisons de ce choix. S'agit-il d'une exigence linguistique, d'une exigence littéraire? C'est plutôt que traduire est suspect. Le traducteur recule (est-ce consciemment ou à son insu?) devant certaines audaces, ce qui le conduit à censurer le texte. Il procède à un déguisement de l'original. Mais «la censure traduisante ne porte pas sur n'importe quoi» (Berman, 1985, p. 56). La déformation atteint des significations profondes; c'est ici une affaire d'idéologie qui est supprimée par "la touche littéraire». Le traducteur soucieux de perfection corrige la langue de l'auteur, là où précisément l'intention de l'auteur se loge dans une violation de la grammaire. Et en apportant de la "beauté" au texte, il laisse tomber la signification. Or, le gommage de la signification entraîne l'oblitération du sens. ${ }^{3}$ Le texte en languecible aura réussi à occulter l'intention ${ }^{4}$ de l'auteur en détruisant tout ce travail subtil et minutieux destiné à marquer le texte comme subversif. Dans un texte rendu conformiste, l'emergence du je ne peut se lire que comme coquille ou lapsus.

Université de Montréal

\section{Références}

ANGENOT, Marc (1978), «Fonctions narratives et maximes idéologiques». Orbis Litterarum, $\mathrm{n}^{\circ}$ 33, pp. 95-110.

Austin, John L. (1970). Quand dire, c'est faire. Paris, Seuil.

Benveniste, Émile (1966). Problèmes de linguistique générale I. Paris, Gallimard.

3. Dans le cadre théorique des actes de langage, nous employons - en suivant Austin (1970) - le mot «sens* comme contenu de ce qui est dit. Signification (meaning) désigne le sens plus la référence.

4. Le mot «intention» est utilisé ici dans le sens qu'il prend à l'intérieur de la théorie des actes de langage. Cf. en particulier ce que dit J. Searle à propos des actes illocutionnaires. (J. Searle, 1972, pp. 83-91). 
BERMAn, Antoine (1985). «La Traduction et la lettre, ou l'auberge du lointain».

Les Tours de Babel, Gérard Granel, éd. Mauvezin, Trans-Europ-Repress, pp. 31-150.

Blanchot, Maurice (1969). L'Entretien infini. Paris, Gallimard.

Carpentier, Alejo (1975a). Le Recours de la méthode. Paris, Gallimard. (1975b). El Recurso del Método. Buenos Aires, Siglo XXI.

SEARLE, John R. (1972). Les Actes de langage. Paris, Hermann. 